\title{
TRES NUEVAS ESPECIES DEL SUBGÉNERO DISTEPHANA (JUSS) KILIPP (PASSIFLORACEAE) PARA RONDÔNIA, BRASIL Especies del Subgénero Distephana para Rondônia
}

\author{
THREE NEW SPECIES OF SUBGENUS DISTEPHANA (Juss) KILIPP (Passifloraceae) \\ for Rondonia, Brasil Species of Subgenus Distephana Rondônia \\ Diego Geraldo Caetano Nunes ${ }^{1}$, Santina Rodrigues Santana, Creucí Maria Caetano² \\ ${ }^{1}$ Investigador FACIMED (Faculdade de Ciências Biomédicas de Cacoal, Brasil), \\ ${ }^{2}$ Docente Investigadora UNAL Palmira
}

Recibido: Julio 1 de 2010

Aceptado: Noviembre 30 de 2010

Correspondencia: Universidad Nacional de Colombia sede Palmira, Edificio de Posgrados: secretaria, Cr 32 no. 12-00, Chapinero vía Candelaria, Palmira Valle Del Cauca, dgcaetanon@unal.edu.co

\section{RESUMEN}

Se describen tres nuevas especies del subgénero Distephana (género Passiflora) para el Estado de Rondônia, amazonia brasileña: Passiflora glandulosa Cav, P. tholozanii Sacco e P. vitifolia Kunth. Estas, sumándose a las anteriormente descritas P. coccinea Ambl., P. araujoi Sacco e P. variolata Poep \& Endl., señalan la diversidad del subgénero en esta región, representado por pocas especies dentro del país.

Palabras clave: género Passiflora, Amazonia brasileña, subgénero Distephana, supersección Distephana.

\begin{abstract}
We are describing three new species of subgenus Distephana (genus Passiflora) to Rondônia State, in the Brazilian Amazonia: Passiflora glandulosa Cav, P. tholozanii Sacco and P. vitifolia Kunth. In addition to the early described, $P$. coccinea Ambl., P. araujoi Sacco and P. variolata Poep \& Endl., presence of these new species points to the diversity of the subgenus in this region, which is represented by a low number of species through Brasil.
\end{abstract}

Key words: genus Passiflora, Brazilian Amazonia, subgenus Distephana, super section Distephana.

\section{INTRODUCCIÓN}

Las plantas del subgénero Distephana (Passiflora L.), según Killip (1) se caracterizan como herbáceas o enredaderas leñosas; flores con color rojo muy intenso; brácteas verticiladas con glándulas en los márgenes, altamente variables en tamaño y forma; cáliz corto y cilíndrico, raramente campaneado, más pequeño que los sépalos; corona en 2 (dos) o 3 (tres) series, interna con fimbrias; membrana tubular, opérculo pendiente; ovario sub-trígono, afilando para los estilos, que son distintos ligeramente unidos en la base; algunas veces ovario sub-truncado y estilos proyectándose desde el ápice de los ángulos, de esta manera similar al subgénero Astrophea Killip. En reciente revisión, Feuillet \& McDougal (2) reclasifican el subgénero como una supersección del género Passiflora.

Particularmente para Brasil, Cervi y Dunaiski Jr. (3) reportan diez especies del subgénero Distephana y sus distribuciones en los Estados. En Colombia, el país que presenta el mayor número de pasifloráceas descritas, se citan seis especies, especialmente en la región amazónica Ocampo et al.(4). Entre las especies descritas para Brasil, se reporta la ocurrencia de tres para el Estado de Rondônia, en la Amazonia brasileña: P. coccinea Ambl., P. araujoi Sacco y P. variolata Poep \& Endl.

En la presente nota se registra la ocurrencia de tres

Rev. Invest. Univ. Quindío (21): 67- 72. Armenia - Colombia 
nuevas especies del subgénero Distephana para Rondônia, Passiflora glandulosa Cav, P. tholozanii Sacco y $P$. vitifolia Kunth, esta última el segundo registro para Brasil.

\section{MATERIALES Y MÉTODOS}

Las colectas y observaciones del material botánico se realizaron en los municipios de Alto Alegre, Cacoal y Pimenta Bueno, Estado de Rondônia, en la región amazónica brasileña (Figura 1).

El municipio de Alto Alegre dos Parecis está localizado en la región sudoeste de Rondônia, con una área geográfica correspondiente a $3.938 \mathrm{~km}^{2}$. El clima de la región se clasifica como caliente y húmedo, con estación seca bien definida, tipo AW según Koppen. La porción norte de este municipio está localizada en la Chapada dos Parecis, que compone la parte final del Planalto Central Brasileño, a altitud media de 500 m.s.n.m. Siguiendo al extremo sur de la región está la planicie o calla del río Guaporé. La vegetación es diversa, presentando en la Chapada dos Parecis vegetación tipo amazónica bien comprometida, en el lado sur de la misma una vegetación más escasa y poco variada.

El municipio de Cacoal está localizado en la región centro-sur, con una área geográfica de $3.808,5 \mathrm{~km}^{2}$. El clima es del tipo tropical caliente y húmedo con dos estaciones: verano lluvioso e invierno seco. El relieve plano y suavemente ondulado, las altitudes mayores no ultrapasan 500 m.s.n.m. La vegetación predominante en la región es de foresta densa, del tipo amazónica, y una pequeña parte de sabana, actualmente reducidas con el proceso de colonización (5).

El municipio de Pimenta Bueno está localizado en la porción sudeste del Estado y ocupa una extensión territorial de $6258,0 \mathrm{~km}^{2}$. El clima de la región se caracteriza como amazónico, ecuatorial y húmedo, y se diferencia por una estación seca. Relieve con terrenos más planos en la mitad norte del municipio, al sur con superficies tabulares so-erguidas sobre terrenos sedimentares, y en el extremo nordeste una área más pronunciada y densa. La vegetación original en grande parte fue removida en el proceso de ocupación humana, los tipos encontrados son foresta amazónica y sabana habiendo una transición entre estos (6).

El período de colectas ocurrió entre febrero de 2005 y agosto de 2007. Los instrumentos utilizados para colecta del material botánico fueron la prensa, pié de rey, lupa, GPS para registro de las coordenadas geográficas y máquina fotográfica digital. Una vez caracterizado, cada especímen completo sufrió el proceso de deshidratación en horno, seguido de choque térmico en un "freezer", retornando al horno por otro período. Las exsicatas se prepararon según las técnicas convencionales e identificadas. Estas se depositaron en el herbario de la FACIMED (Faculdade

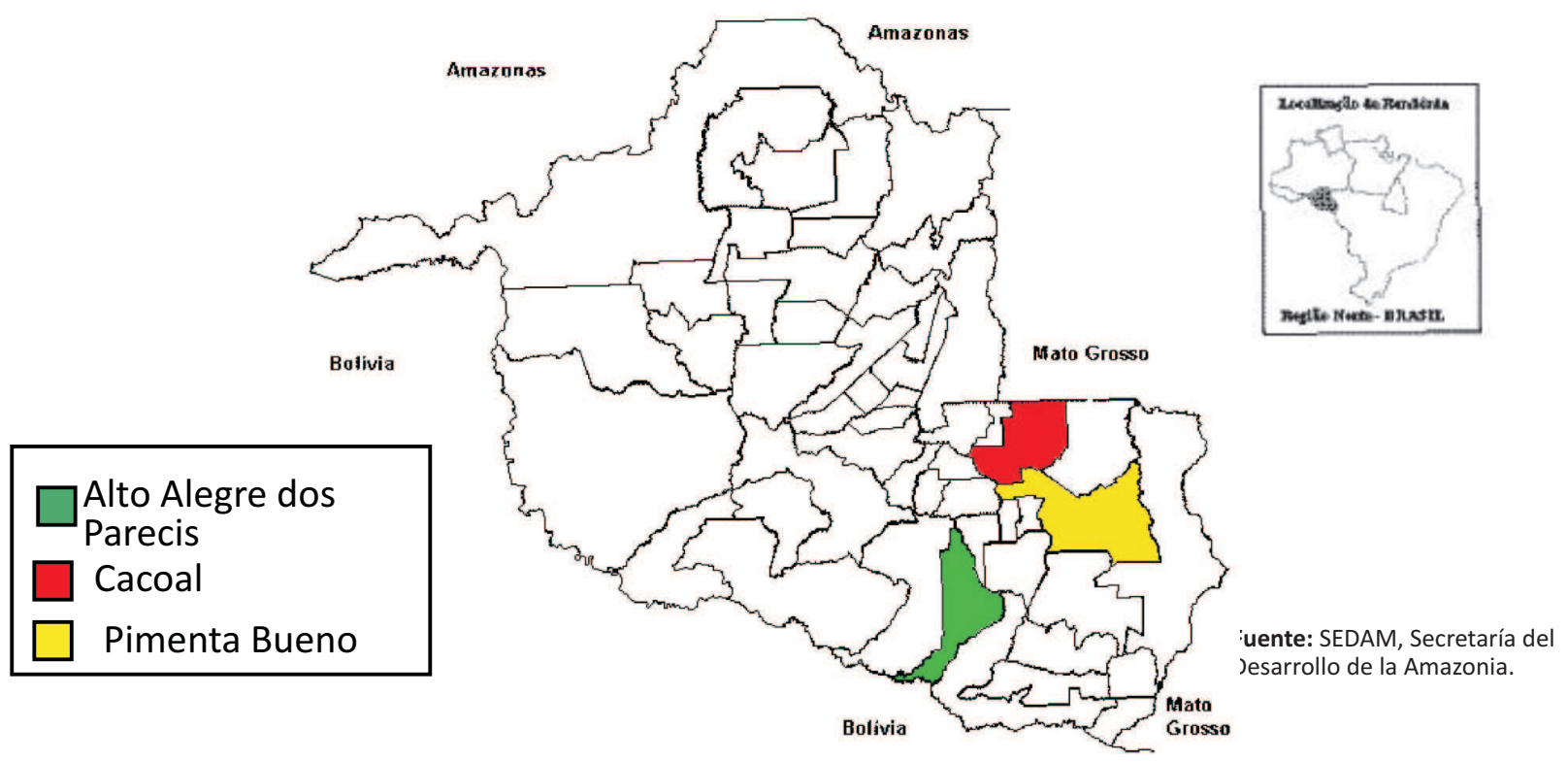

Figura 1. Mapa de los municipios rondonienses en estudio.

Rev. Invest. Univ. Quindío (21): 68- 73. Armenia - Colombia 
de Ciências Biomédicas de Cacoal, Brasil), siendo encaminado un duplicado al herbario $\mathrm{CH}$ de la UFMT (Universidade Federal do Mato Grosso, Cuiabá, Brasil).

\section{RESULTADOS Y DISCUSIÓN}

\section{P. glandulosa}

Planta enredadera o liana (Figura 2), de rara ocurrencia en el municipio de Pimenta Bueno. La descripción general concuerda con Killip (1) y Cervi y Dunaiski Jr (3), excepto por el tallo cilíndrico, con algunas estrías transversales; pecíolo de 9-15 mm con dos nectarios; hojas oblongo-lanceoladas o lanceoladas de 84-110 x 30-40 mm; pedúnculo de $25-60 \mathrm{~mm}$; sépalos mucronados con $42-46 \times 9 \mathrm{~mm}$, con dos pares de glándulas; pétalos $46 \times 9 \mathrm{~mm}$; corona de filamentos compuesta por 2 series, externa 10-13 mm, ovario 5-6 $\times 3 \mathrm{~mm}$.

Distribución para el Brasil: Amazonas, Pará, Acre,

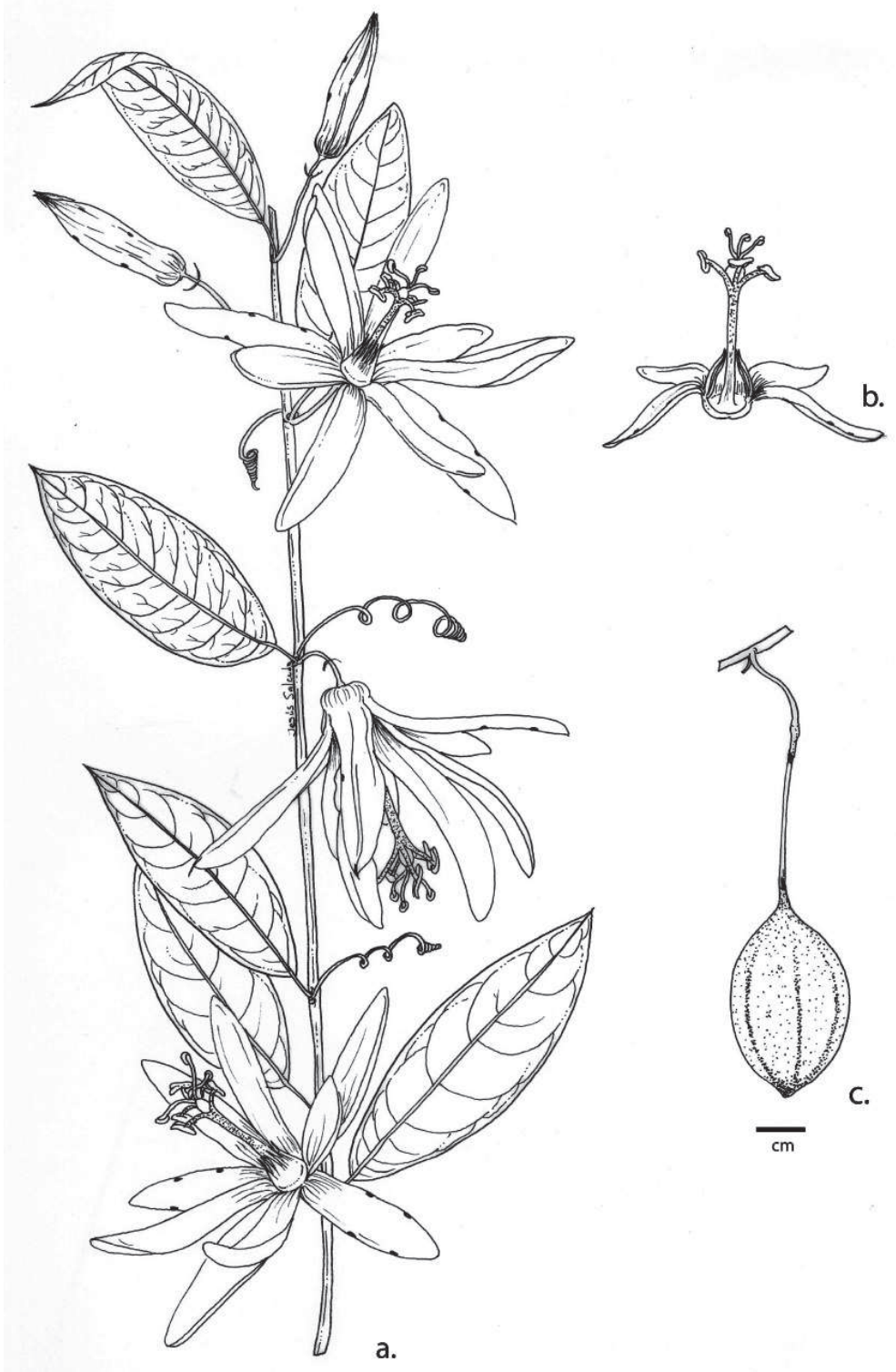

Figura 2. P. glandulosa Cav.

Rev. Invest. Univ. Quindío (21): 67- 72. Armenia - Colombia 
Amapá, Roraima, Maranhão, Ceará, Paraíba, Rio de Janeiro (cultivada) Cervi y Dunaiski Jr, (3), y el primero registro para Rondônia.

Fenología: Florece en junio y fructifica en agosto.

\section{P. tholozanii}

Planta enredadera (Figura 3), abundante en el municipio de Alto Alegre. Diferenciase de la descripción de Killip (1) y Cervi y Dunaiski Jr (3) por el tallo cilíndrico con algunas estrías transversales, levemente pubescente; estipulas lineares de 3-5 mm de longitud; pecíolos de 15-18 mm de longitud con 2 glándulas en la base; hojas de 70-120 x 40-80 mm, con glándulas de néctar en sus márgenes, superficie adaxial levemente pubescente, abaxial un poco más pubescente; pedúnculo $72 \mathrm{~mm}$; sépalos 49-52 x 10-12 $\mathrm{mm}$; pétalos 47-51 x 10-14 mm; corona de filamentos con 2 series, la externa con filamentos de $13-15 \mathrm{~mm}$, interna de 10-13mm; ovario 7-9mm.

Distribución para el Brasil: Amazonas, Pará, Amapá,

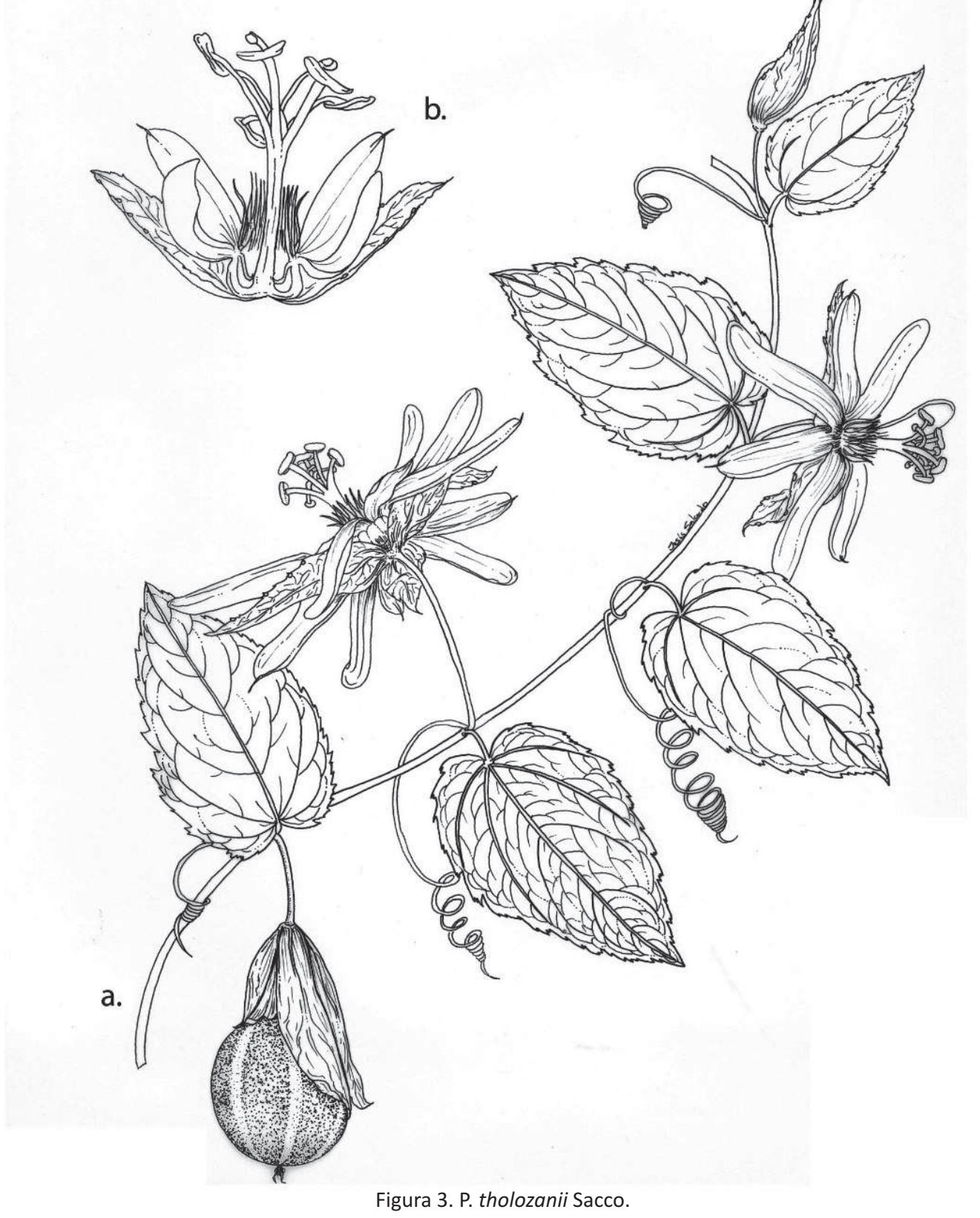

Rev. Invest. Univ. Quindío (21): 68- 73. Armenia - Colombia 
Mato Grosso Cervi y Dunaiski Jr, (3), y el primero registro para Rondônia.

Fenología: Florece en agosto y fructifica en septiembre.

\section{P. vitifolia}

Planta enredadera (Figura 4), de rara ocurrencia en el municipio de Cacoal. Diferenciase de la descripción de Killip (1) y Cervi y Dunaiski Jr (3) por el tallo cilíndrico levemente sub-angular, poco pubescente; pecíolo de
14-20 mm de longitud con 5 a 9 nectarios dispersos; hojas trilobuladas de 98-112 x 92-117 mm; pedúnculo 5-7 mm; brácteas 10-20 x 3-6mm; sépalos rojos de 60$62 \times 11-18 \mathrm{~mm}$, quilla en color verde; pétalos de 50-56 x 9-12 mm; corona con 3 series de filamentos color rojo, con pigmentación blanca en la base; filamentos externos $21 \mathrm{~mm}$, medianos $13 \mathrm{~mm}$ e internos $9 \mathrm{~mm}$.

Distribución para el Brasil: Amazonas Cervi y Dunaiski Jr, (3), y el primero registro para Rondônia.

Fenología: florece en febrero y fructifica en marzo.

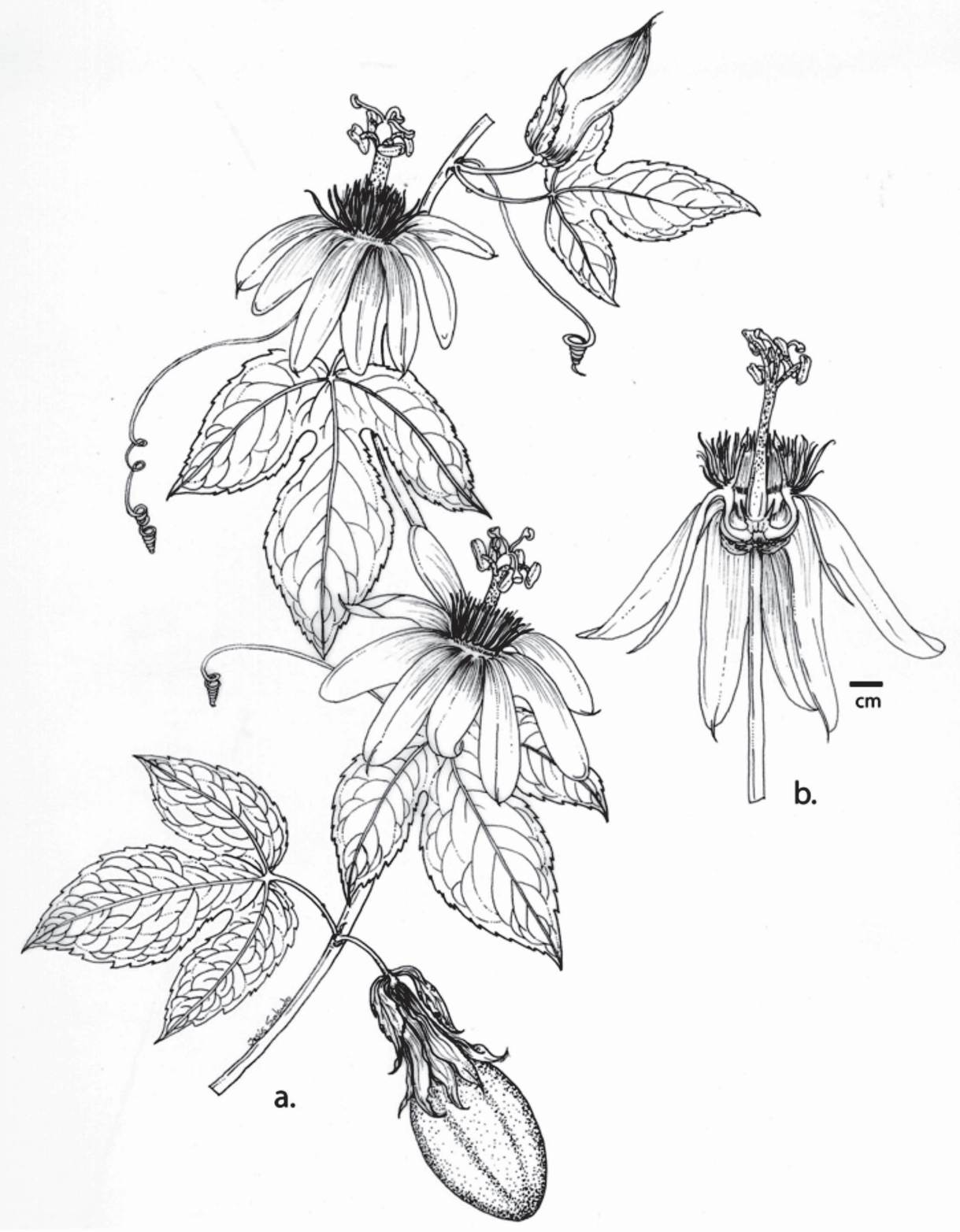

Figura 4. P. vitifolia Kunth. 


\section{CONCLUSIÓN}

El subgénero Distephana está bien representado en el Estado de Rondônia, donde ocurren seis de las 10 especies reportadas para el país: P. araujoi, $P$. coccinea, $P$. glandulosa, P. tholozanii, P. variolata y P. vitifolia.

\section{AGRADECIMIENTOS}

Al profesor Dr. Armando C. Cervi, por colaborar con la identificación de las especies.

\section{BIBLIOGRAFÍA}

1. Killip, E. P. (1938). The American species of Passifloraceae. Chicago: Field Museum Natural History Publication, Botanical Series, 19 (1, 2): 1-613.

2. Feuillet, C. y McDougal, J.M. (2003). A new infrageneric classification of Passiflora L. (Passifloraceae). Passiflora, 13(2): 34-38.

3. Cervi, A. C. y Dunaiski Jr, A. (2004). Passifloraceae do Brasil: Estudo do gênero Passiflora L. subgênero Distephana (JUSS) KILLIP, Revista Estudos de Biologia, 26 (55): 45-67.

4. Ocampo P., J., Coppens d'Eeckenbrugge, G., Restrepo, M., Jarvis, A., Salazar, M. y Caetano, C. (2007). Diversity of Colombian Passifloraceae: biogeography and an updated list for conservation. Biota Colombiana, 8 (1): 1-45.

5. Kemper, L. (2006). Cacoal sua história sua gente. Goiânia, Grafopel, 2ำed., 240p.

6. Almicar, A. (2005). Zoneamento geoambiental de Pimenta Bueno. Disertación de Maestría. Universidade Federal de Rondônia-UNIR Núcleo de Ciências e Tecnologia. 